Ann. Biol. anim. Bioch. Biophys., I970, 10 (2), 301-308.

\title{
DÉTECTION DE LA TYLOSINE DANS LES TISSUS ANIMAUX
}

\author{
Paulette VALDEBOUZE, Fernande BOZZI et Danielle FRÉRES \\ avec la collaboration technique de Françoise BaLland \\ Laboratoire d'Essai et d'Analyse des Aliments, \\ 1, Rue Santos-Dumont, Paris (I $5^{\mathrm{e}}$ ) \\ Institut national de la Recherche agronomique
}

RÉSUMÉ

Grâce à une méthode améliorée de détection de la tylosine dans les tissus animaux, il a été confirmé qu'aucun des principaux tissus de porcs ayant reçu, durant toute leur croissance, un aliment à Ioo p.p.m. (1) de tylosine, ne retient plus de 0,4 p.p.m. de cet additif (limite de détec. tion).

\section{INTRODUCTION}

L'addition de tylosine à la ration des porcs a un effet favorable sur leur croissance (JoRdAN et al., I960, I96r). Cependant, l'utilisation des antibiotiques en alimentation animale ne doit s'accompagner d'aucune accumulation de résidus susceptibles de provoquer chez l'homme des phénomènes d'allergie ou l'apparition de souches microbiennes résistantes.

FERRANDO (I968), faisant mention d'un travail non publié de BERKMAN et VAN DUYN (r964), indique que l'ingestion de tylosine par le porc ne provoquerait pas 1'apparition de résidus dans ses tissus même après 1'emploi de doses élevées.

Après avoir procédé à la mise au point d'une méthode sensible de détection de la tylosine dans divers tissus de porc, nous avons, dans le présent travail, tenté de mesurer l'activité antibiotique résiduelle dans les tissus d'animaux ayant reçu durant quatre mois, une dose élevée de tylosine.

(1) p.p.m. = parties par million. 


\section{I. - ÉTUDE CRITIQUE DE IA MÉTHODE}

Tout en gardant le principe du dosage microbiologique par diffusion dans la gélose ensemencée par Sarcina lutea A. 'T. C. C. 934I (KLINE et al., I965), nous avons choisi d'autres solvants d'extraction que ceux proposés par ces auteurs, préféré aux cylindres les disques de papier-filtre imprégnés de l'extrait et déposés sur la gélose ensemencée; nous avons également retenu une définition différente de la sensibilité de la méthode. Ces modifications sont incluses dans la méthode définitive décrite en annexe.

\section{Détermination du seuil de sensibilité de la méthode}

La sensibilité d'une méthode de détection de résidus à activité antibiotique est très souvent déterminée de la façon suivante : une gamme étalon est préparée par dilution d'une solution d'antibiotique dans un extrait de tissu témoin. La plus faible concentration qui donne une zone d'inhibition sert à calculer la dose limite d'antibiotique pouvant être détectée.

TABLEAU I

Taux de recouvrement de tylosine ajoutée à du muscle de porc par rapport à celui d'un étalon préparé à partir d'extrait témoin

\begin{tabular}{c|c|c}
\hline $\begin{array}{c}\text { Quantité ajoutée } \\
\text { (mcg/g) }\end{array}$ & $\begin{array}{c}\text { Taux de recouvrement } \\
\text { (p. 100) }\end{array}$ & $\begin{array}{c}\text { Solvant } \\
\text { d'extraction }\end{array}$ \\
\hline 1,6 & $\begin{array}{c}89-85-633 \\
89-87\end{array}$ \\
1,2 & $91-79-87-110$ & Eau \\
1,0 & $76-87-97$ & Méthanol \\
0,7 & & - \\
\hline
\end{tabular}

TABLEAU 2

Taux de vecouvrement de tylosine ajoutée à des tissus de porc par rapport à celui d'un étalon préparé à partir d'un extrait témoin (KLINE, 1967)

\begin{tabular}{|c|c|c|}
\hline Tissus & $\begin{array}{l}\text { Quantité de tylosine } \\
\text { ajoutée en } \mathrm{mcg} / \mathrm{g}\end{array}$ & $\begin{array}{c}\text { Taux de recouvrement } \\
(\text { p. 100) }\end{array}$ \\
\hline \multirow{2}{*}{ Foie } & 0,3 & 53 \\
\hline & 0,5 & 52 \\
\hline \multirow{2}{*}{ Muscle } & 0,3 & $81-69$ \\
\hline & 0,5 & $96-99$ \\
\hline \multirow{2}{*}{ Graisse } & 0,1 & 97 \\
\hline & 0,3 & 82 \\
\hline \multirow{2}{*}{ Rein } & 0,3 & 130 \\
\hline & 0,5 & 126 \\
\hline
\end{tabular}


Cette technique est différente de celle pratiquée lors du dosage lui-même et peut conduire à des résultats erronés.

En effet, lors de la préparation de l'échantillon, le tissu et l'antibiotique qu'il contient sont extraits ensemble par le solvant. En effectuant le dosage de l'antibiotique, ajouté en quantités connues à des tissus témoins avant extraction, et en comparant ces échantillons à ceux d'une gamme préparée à partir d'un extrait de tissu témoin, nous avons constaté que les taux de recouvrement sont souvent très éloignés de Ioo, en particulier pour les faibles doses (tabl. I).

KLINE (I967) a obtenu, pour le même antibiotique, des résultats encore plus significatifs; il en utilisait, il est vrai, des doses plus faibles (tab1. 2).

Nous préférons donc, pour notre part, déterminer la sensibilité de la méthode en préparant les solutions étalons dans les mêmes conditions que les échantillons. Pour cela, des quantités connues d'antibiotique sont ajoutées à des tissus témoins avant extraction. La plus faible concentration d'antibiotique, donnant des zones d'inhibition parfaitement lisibles, indique la limite de sensibilité.

\section{Choix du solvant d'extraction}

KLINE (I967) utilise l'eau comme agent d'extraction. Nous avons préféré employer, suivant les tissus, le méthanol pur ou un mélange méthanol-tampon $\mathrm{pH} 8$. Ces deux bons solvants de la tylosine, plus volatils que l'eau, conduisent à une concentration plus poussée sur les disques de papier-filtre et, de ce fait, à une amélioration de la sensibilité. En outre, ils facilitent la séparation des phases solide et liquide lors de la centrifugation des extraits.

Pour le muscle, nous avons choisi un mélange à parties égales de méthanol et de tampon phosphate-bicarbonate à $\mathrm{pH}$ 8. La limite de sensibilité ainsi atteinte est de 0,2 p.p.m. contre o,6 p.p.m. avec le méthanol pur.

Pour le foie, nous avons dû, au contraire, augmenter la proportion de méthanol dans le mélange méthanol-tampon; le solvant organique réduit la quantité de substances extraites en même temps que l'antibiotique, qui gênent la concentration de l'extrait sur les disques de papier. Nous avons obtenu une sensibilité de o,2 p.p.m. en utilisant un mélange de méthanol et de tampon dans la proportion 8o/2o et une sensibilité de 0,4 p.p.m. avec ce même mélange dans la proportion 50/50.

Enfin, dans le cas du sang, nous avons dû faire appel au méthanol pur.

\section{Emploi de disques de papier}

Nous avons choisi d'imprégner en plusieurs fois des disques de papier-filtre par les extraits de tissus, ainsi qu'il est préconisé par Pitre et Obaton (r964). La concentration des extraits est ainsi facilitée et le disque surchargé peut être mis directement en contact avec le germe test.

Cette technique ne requiert que des moyens limités et rend possible l'exécution de grandes séries de dosages.

\section{Zones d'inhibition non spécifiques}

Certains auteurs (PEDERsen, I965; FERrando, r968) ont signalé la présence de zones d'inhibition non spécifiques lors du dosage des antibiotiques dans les tissus d'animaux témoins. Ces zones pourraient être dues à des substances antibactériennes 
d'origine cellulaire (Skarnes et Watson, I957 ; Solarino, I96I ; DELAUNAy, I96r) extraites par le solvant utilisé. Dans nos conditions opératoires, nous n'avons jamais observé la présence de telles zones à partir de divers tissus de porcs témoins.

\section{II. - APPLICATION A LA DÉ'TECTION DE RÉSIDUS}

\section{A ACTIVITÉ, ANTIBIOTIQUE DANS IES TISSUS DE PORC}

I a méthode telle qu'elle est décrite en annexe a été appliquée à la détermination d'un seuil de sensibilité de la détection de l'antibiotique dans chaque tissu analysé.

Les résultats obtenus au cours de dosages répétés d'antibiotique ajouté à des tissus d'animaux témoins nous conduisent à proposer les limites de sensibilité suivantes :

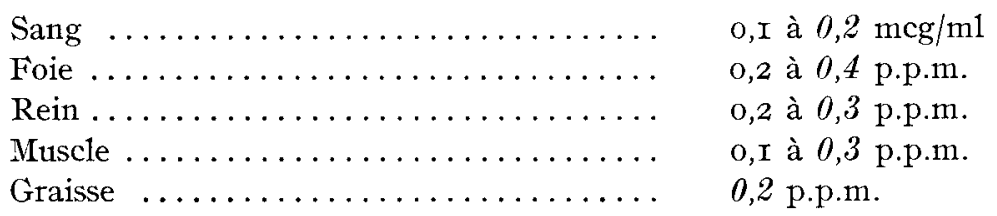

La valeur la plus élevée est seule retenue comme seuil de détection de l'antibiotique dans le tissu considéré.

D'autre part, la mise en évidence de résidus à activité antibiotique a porté sur les tissus de dix porcs mâles castrés soumis à un régime antibiosupplémenté. Ceux-ci ont reçu, durant une période de quatre mois, un aliment composé de :

\begin{tabular}{|c|c|}
\hline Orge & $7^{2} \mathrm{p} .100$ \\
\hline Son $\ldots \ldots \ldots$ & Io p. IOO \\
\hline Tourteau de soja & I2 p. IOO \\
\hline Composé minéral . ......... & 2 p. IOO \\
\hline $\begin{array}{l}\text { Prémélange orge }+ \text { vitamines } \ldots \ldots \ldots \ldots \ldots \ldots \\
\text { Tylosine (incorporée par 1'intermédiaire }\end{array}$ & 4 p. IOo \\
\hline
\end{tabular}

L'aliment a été distribué à raison de $400 \mathrm{~g}$ de matière sèche par Io $\mathrm{kg}$ de poids vif jusqu'à $70 \mathrm{~kg}$ et $350 \mathrm{~g}$ de matière sèche par to $\mathrm{kg}$ de poids vif au-delà de ce poids. Deux animaux servant de témoins ont reçu le même aliment sans antibiotique. Les animaux ont été abattus à un poids compris entre 90 et $100 \mathrm{~kg}$. Les tissus prélevés pour l'analyse (muscle, foie, rein, graisse, sang) ont été conservés à $+4^{\circ} \mathrm{C}$ durant le temps précédant l'analyse (maximum 6 jours).

Chez ces porcs ayant reçu dans leur aliment cinq fois la dose normale de tylosine, il n'est apparu aucune activité antibiotique supérieure aux limites de sensibilité de la méthode.

Il faut cependant noter que cette dernière ne dose que les résidus à activité antibiotique extraits par le méthanol ou un mélange méthanol-tampon. Certaines formes " liées " et tous les produits de dégradation qui n'ont plus d'activité antibio- 
tique lui échappent. Cette critique n'est pas spécifique de la présente méthode ; elle marque la limite d'une technique intéressante, par ailleurs, par son caractère biologique et sa sensibilité.

Reçu pour publication en novembre 1969.

\section{REMERCIEMENTS}

Ce travail a été réalisé en partie grâce à la collaboration financière de la firme Eli-Lilly à laquelle nous adressons nos remerciements.

\section{SUMMARY}

\section{DETECTION OF TYLOSIN IN PIG TISSUES}

A method has been developed for the microbiological detection of tylosin in pig tissues. Extracts obtained from the tissues are concentrated on paper filter discs. Their antibiotic activities were demonstrated by diffusion in an agar medium inoculated with Sarcina lutea A. T. C. C. 934I.

When applied to the analysis of various tissues of pigs receiving a feed containing roo p.p.m. of tylosin for 4 months, this method shows that there are no residues having an antibiotic activity higher than $0.2 \mathrm{mcg} / \mathrm{ml}$ for the blood, 0.4 p.p.m. for the liver, 0.3 p.p.m. for the kidney and the muscle, and 0.2 for the fat. These levels correspond to the limits of sensitivity of the method.

\section{RÉFÉRENCES BIBLIOGRAPHIQUES}

Berkman R. N., Van Duyn R. L., I964. Lilly pharm. exper. in Ferrando R., i968, ci-dessous.

Delaunay A., I96I. Substances bactéricides (ou bactériostatiques) présentes dans l'organisme des mammifères. In Aux frontières de la microbiologie médicale II/cahier 65-84, Flammarion, Paris.

Ferrando R., 1968. Absorption et métabolisme des antibiotiques. Résidus dans les tissus. Antibiotiques en agriculture. Nutr. Dieta, 10,90-125.

Jordan C. E., Waits W. P., Means T. M., I960. Tylosin. A new antibiotic growth stimulant for swine. (abstr.). J. anim. Sci., 19, I269.

Jordan C. E., Waitt W. P., Means T. M., Carter F. R., Redman C. E., ig6r. Comparison of three tylosin feeding regimes for growing-finishing swine. (abstr.). J. anim. Sci., $20,937$.

KLINE R. M., I967. Données non publiées.

KLINe R. M., Redman C. E., Tepe J. B., Hamill R. L., I965. Antibiotic residues. Microbiological procedures. Agric. Food Chem., 13, 301-303.

Pedersen H., I965. The use of concentrated extracts from tissues in the determination of penicillin and tetracyclins in slaughtered poultry to which fooders containing antibiotics have been fed. $\mathrm{Kgl}$. Vet. Landbohoyskole, A. R. S. S. K., 33-60.

Pitre J., Obaton J., I964. Accroissement de la sensibilité de la méthode des disques par imprégnations répétées pour la détection et le titrage des antibiotiques présents dans le lait, les viandes et les aliments du bétail. Bull. Ac. Vét. France, 37, 63-69.

Skarnes R. C., Watson D. W., I957. Antimictobial factors of normal tissues and fluids. Bacteriol. Rev., 21, 273-294.

Solarıno G., Ig6r. Antibiotiques endogènes d'origine tissulaire. Bull. Acad. Suisse Sci. Mêd., 17,43 I. 


\section{ANNEXE \\ MÉTHODE DE DOSAGE DE LA TYLOSINE DANS LES TISSUS DE PORC}

\section{Objet et domaine d'application}

Cette méthode s'applique au dosage de la tylosine dans le muscle, le foie, le rein, la graisse et le sang de porc.

\section{Principe}

La tylosine est extraite par le méthanol pur ou un mélange méthanol-tampon phosphate bicarbonate $\mathrm{pH}$ 8. L'activité antibiotique des extraits, concentrés sur disques de papier-filtre est mesurée sur Sarcina lutea A. T. C. C. 934 I par diffusion en milieu gélosé.

\section{Milieux de cultuve}

3. I. Milieu d'entretien du germe.

\begin{tabular}{|c|c|}
\hline Peptone ... & 6 \\
\hline Casitone ............ & 4 \\
\hline Extrait de levure..$\ldots \ldots \ldots$ & 3 \\
\hline Extrait de viande de bouf ... & $\mathrm{I}, 5$ \\
\hline 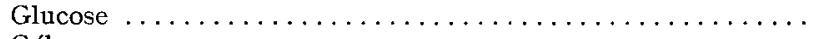 & I \\
\hline 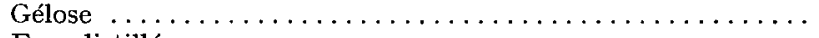 & I 5 \\
\hline Eau distillée q.s.p. & 000 \\
\hline
\end{tabular}

Le $\mathrm{pH}$, après stérilisation de 20 minutes à $\mathrm{I} 20^{\circ} \mathrm{C}$, doit être compris entre 6,5 et 6,6 .

3. 2. Milieu de dosage ( $\mathrm{r}$ ere couche).

\begin{tabular}{|c|c|}
\hline Peptone & 5 \\
\hline Extrait de viande de bouf & 1,5 \\
\hline Extrait de levure $\ldots \ldots \ldots \ldots \ldots$ & $\mathbf{I}, 5$ \\
\hline Chlorure de sodium $\ldots \ldots \ldots \ldots$ & 3,5 \\
\hline Gélose & I 5,0 \\
\hline Eau distillée q.s.p. & I ooo \\
\hline
\end{tabular}

Ajuster le pH à 8,3 avant stérilisation. Stériliser 30 minutes à $\mathrm{I} 20^{\circ} \mathrm{C}$.

3. 3. Milieu de dosage ( $2^{\mathrm{e}}$ couche).

Même composition que le milieu 3.2, mais avec addition de Io $\mathrm{g}$ de gélose pour I ooo $\mathrm{m}$ au lieu de $5 \mathrm{~g}$.

\section{Solvants}

4. I. Méthanol pur.

4. 2. Tampon phosphate-bicarbonate pH 8 .

Phosphate dipotassique .................. I6,73 g

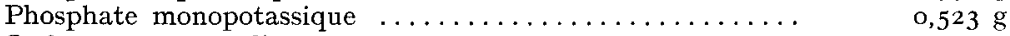

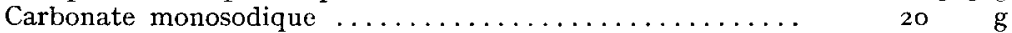

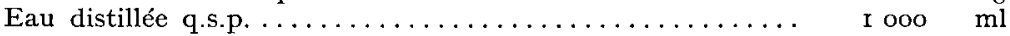

4. 3. Mélange méthanol-tampon no 1.

Méthanol pur ........................ 50 $\mathrm{ml}$

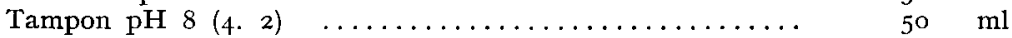

4. 4. Mélange méthanol-tampon no 2.

Méthanol pur

$80 \mathrm{ml}$

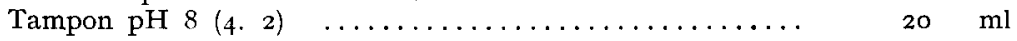


4. 5. Solution de chloruve de sodium.

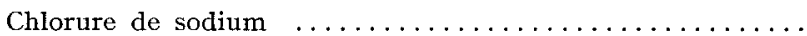

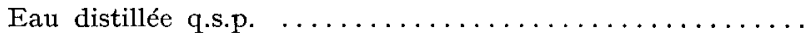

${ }_{\mathrm{I} \text { ooo }}^{9, \mathrm{I} 2 \mathrm{~g}} \mathrm{ml}$

4. 6. Solution de citrate de sodium.

Citrate trisodique cristallisé

I $\quad \mathrm{g}$

Solution de chlorure de sodium (4. 5) q.s.p.

1000

ml

\section{Microorganisme}

Sarcina lutea A. T. C. C. 934 I.

5. I. Conservation de la souche mère.

Inoculer $S$. lutea sur milieu d'entretien (3. I). Incuber 48 heures à l'étuve à $30^{\circ} \mathrm{C}$. Conserver au réfrigérateur entre 4 et $10^{\circ} \mathrm{C}$. Entretenir cette souche par repiquage hebdomadaire sur gélose inclinée.

\section{2. Préparation de la suspension de germes.}

Récolter les germes d'une culture de $4^{8}$ heures (5. I) par Io $\mathrm{ml}$ de sérum physiologique stérile. La densité optique (Coleman junior) de la suspension ainsi obtenue doit être de 0,9 à $630 \mathrm{~nm}$. Déterminer, par des essais préliminaires, la quantité d'inoculum nécessaire pour obtenir, avec les concentrations d'antibiotique utilisées, des zones d'inhibition aussi grandes et aussi nettes que possible.

L'inoculation du milieu de culture se fait à $4^{80} \mathrm{C}$. Conservée à $+4^{\circ} \mathrm{C}$, cette suspension peut être utilisée pendant I 5 jours.

\section{Préparation des échantillons}

6. 1. Sang.

Recueillir le sang dans des flacons de I ooo $\mathrm{ml}$ contenant $\mathbf{I} 50 \mathrm{ml}$ de solution de citrate de sodium (4. 6), stérilisés 20 minutes à $120^{\circ} \mathrm{C}$. Le conserver à $+4^{\circ} \mathrm{C}$.

6. 2. Muscle, graisse, foie, rein.

Débarrasser les muscles des nerfs et de la graisse, les découper et les broyer au hachoir à viande. Traiter la graisse de la même manière. Découper simplement au couteau le foie et le rein. Conserver les prises d'essais convenables dans des sacs de matière plastique, à $+4^{\circ} \mathrm{C}$, en attendant l'analyse.

\section{Extraction}

\section{I. Muscle.}

Peser $50 \mathrm{~g}$ de tissu. Ajouter $50 \mathrm{ml}$ de mélange méthanol-tampon no I (4. 3). Broyer à l'homogénéisateur. Centrifuger 20 minutes à 9 ooo tours $/ \mathrm{mn}$ à $+4^{\circ} \mathrm{C}$.

\section{2. Foie, rein et graisse.}

Peser 50 g de tissu. Ajouter $50 \mathrm{ml}$ de mélange méthanol-tampon $\mathrm{n}^{\circ} 2$ (4. 4). Broyer à l'homogénéisateur. Centrifuger 20 minutes à 9 ooo tours $/ \mathrm{mn}$ à $+4^{\circ} \mathrm{C}$.

\section{3. Sang.}

Introduire $25 \mathrm{ml}$ de sang dans un tube à centrifuger. Ajouter $25 \mathrm{ml}$ de méthanol pur (4. $\mathrm{r}$ ). Agiter I à 2 minutes. Centrifuger 20 minutes à 9000 tours $/ \mathrm{mn}$ à $+4^{\circ} \mathrm{C}$.

\section{Solutions étalons}

Broyer, pour chaque concentration de la gamme, un tissu témoin additionné de tylosine en solution dans le méthanol pur (4. I), le mélange méthanol-tampon (4. 3) ou (4. 4), suivant les cas, en respectant les proportions tissu-solvant utilisées pour l'échantillon.

Préparer ainsi les concentrations $0,05-0,1-0,2-0,4-0,8 \mathrm{mcg} / \mathrm{ml}$ et un échantillon de tissu témoin. Il représente le point o de la gamme et permet de vérifier que le tissu n'a aucune activité antibiotique par lui-même.

Centrifuger 20 minutes à 9000 tours $/ \mathrm{mn}$ à $+4^{\circ} \mathrm{C}$. 


\section{Concentration des extraits}

Le surnageant des extraits à doser et des solutions étalons est déposé sur des disques de papier filtre, par additions répétées de II gouttes chaque fois, jusqu'à un total de XVI gouttes. Les disques ont été préalablement préparés sur des toiles en acier inoxydable placées sur des boîtes de Petri, afin d'améliorer le séchage, accéléré d'autre part par ventilation et chauffage modéré de la paillasse.

\section{Io. Préparation des boîtes de Petri}

Io. x. Première couche.

Introduire dans chaque boîte de Petri ro $\mathrm{ml}$ de milieu de dosage (3. 2).

Io. 2. Deuxième couche.

Inoculer le milieu de dosage (3.3) par la suspension de germes (5. 2). Répartir à raison de $4 \mathrm{ml}$ par boîte de Petri. Préparer les boîtes le jour même de leur utilisation et les conserver au réfrigérateur à $+4^{\circ} \mathrm{C}$ jusqu'à l'emploi.

\section{Mise en évidence et mesure de l'activité antibiotique}

Déposer les disques (9) à la surface des boîtes de Petri (ro). Utiliser :

a) pour la gamme étalon, dix boîtes contenant chacune les cinq concentrations de l'étalon et le témoin;

b) pour chaque échantillon, trois boîtes renfermant alternativement trois disques imprégnés de l'extrait du tissu (7) et trois de la solution (8) à $0,2 \mathrm{mcg} / \mathrm{ml}$.

Incuber les boîtes, durant zo heures environ, à $30^{\circ} \mathrm{C}$. Mesurer les diamètres des zones d'inhibition. Tracer la droite de référence en portant en abscisse le logarithme des concentrations et les moyennes des diamètres en ordonnée. Lire sur cette droite la teneur en tylosine $(\mathrm{en} \mathrm{mcg} / \mathrm{ml}$ ) de la solution à doser, en tenant compte de la différence moyenne des diamètres des zones d'inhibition de l'étalon et de l'échantillon.

Calculer la teneur en $\mathrm{mcg} / \mathrm{g}$ du tissu ou en $\mathrm{mcg} / \mathrm{ml}$ du sang en fonction de la prise d'essai et des dilutions. 